\title{
Using Voxel-Based Morphometry to Examine Atrophy-Behavior Correlates in Alzheimer's Disease and Frontotemporal Dementia
}

\author{
Michael P. Lin, Christian Devita, James C. Gee, and Murray Grossman \\ University of Pennsylvania \\ Departments of Radiology and Neurology \\ Philadelphia, PA 19104 \\ gee@grasp.cis.upenn.edu
}

\section{Background}

Computational Morphometry With the advent of more sophisticated computer and medical imaging technology, computational morphometry of MRI images has become a standard tool in the statistical analysis of differences in brain structure between two groups of subjects. This comparative work represents an important advance in the diagnostic armamentarium for neurodegenerative diseases such as Alzheimer's disease (AD) and frontotemporal dementia (FTD), where no definitive diagnostic tests are available. This preliminary report describes morphometric analyses of MRI datasets of AD and FTD patients, and relates these to clinical measures. We will begin this paper by describing voxel based morphometry (VBM), a powerful, quantitative tool for analyzing structural brain images. We then describe our implementation of a fully automated, user-independent algorithm for morphometric analysis of structural MRI data.

Voxel-Based Morphometry (VBM) VBM is a voxel-wise comparison of gray matter intensity, where groups of images are normalized, segmented, smoothed, and then compared using voxel-wise statistical parametric tests. As opposed to label-based approaches to morphometry, where changes are measured over regions of interest (ROI) defined by the operator, VBM does not require the a priori selection of ROIs. This has three main potential advantages over labelbased methods: (a) removal of operator bias in ROI definition (local bias), (b) quantification of subtle features easily missed by operator inspection, and (c) assessment of global structural changes in brain, unrestricted by the selection of specific ROIs (global bias). 11019] One problem with VBM is differences detected due to the presence of mis-segmented, non-brain voxels. These errors become significant as study groups grow large, although algorithms have been developed to manage this problem. [11]

Alzheimer's Disease (AD) AD is perhaps the most common neurodegenerative disease. Yet there is no definitive clinical test for identifying this condition or distinguishing it from other conditions such as FTD. Patients suffering from $\mathrm{AD}$ tend to show symptoms of decreased language aptitude, such as deficits in

T. Dohi and R. Kikinis (Eds.): MICCAI 2002, LNCS 2488, pp. 770-776 2002.

(C) Springer-Verlag Berlin Heidelberg 2002 
naming and semantic comprehension. [12] Areas of atrophy associated with AD may include the temporal-parietal, dorsolateral pre-frontal, and hippocampal regions, based largely on user defined ROI analyses. [17. These areas contribute to language, executive, and memory functioning. [12] It has been claimed that atrophy in the hippocampal formation is a marker for $\mathrm{AD}$, and that this atrophy is related to memory loss. 720] However, this distribution of atrophy may not be specific to $\mathrm{AD}$, as it is also found in temporal lobe epilepsy, schizophrenia and FTD. 9

Frontotemporal Dementia (FTD) Frontotemporal dementia, also known as frontotemporal lobar degeneration or Pick's disease, refers to one important form of non-Alzheimer's dementia. Behavioral studies have shown that patients suffering from FTD have cognitive difficulty, with aphasia (disorder of language), executive resource limitations such as poor working memory and impaired planning, and a disorder of behavior and social comportment. [17] ROI studies suggest that areas of atrophy associated with FTD may include orbitofrontal, dorsolateral pre-frontal, and anterior temporal regions, areas thought to contribute to language and executive resources. [9]

Hypothesis Comparative structural and behavioral studies of AD and FTD have been rare. In our study, we will first use VBM to examine the gray matter structure of FTD and AD patients to determine locations of significant cortical atrophy. Then we will use VBM to look for correlations between regional atrophy and performance on behavioral tests that measure specific cognitive functions. Our hypothesis is that certain types of clinically observable deficits in FTD and $\mathrm{AD}$ patients are due to specific areas of cortical atrophy in the brain, that these brain-behavior relations can be identified through correlation analyses, and that different patterns of brain-behavior correlations in AD and FTD reflect distinct clinical impairments and the unique ways in which complex tasks such as naming can be compromised in different neurodegenerative diseases.

\section{Methods}

Subject Population We studied forty-five subjects in total belonging to one of the patient groups or to a healthy control group. Nine subjects were healthy, elderly controls. The 20 AD patients and 26 FTD patients were diagnosed by a board-certified neurologist using published criteria (see Table 1). All patients were mild to moderately impaired.

Behavioral Tests All of these subjects, in addition to being imaged, performed a battery of cognitive tasks designed to assess their mental ability. The test used to illustrate the correlation analysis in this study was a confrontation naming task known as the "Boston Naming Test" (BNT). The subject is presented with a line drawing of an object and the subject must say the appropriate name for the object.

Magnetic Resonance Imaging Imaging was performed using a GE Horizon Echospeed 1.5 T MRI scanner (GE Medical Systems, Milwaukee, WI). First, a 
Table 1. Subject group breakdown.

\begin{tabular}{|l|c|c|c|c|}
\hline Subject Group & Number & $\begin{array}{c}\text { Mean Age } \\
\text { (St. Dev) }\end{array}$ & $\begin{array}{c}\text { Mean MMSE } \\
\text { (St. Dev) }\end{array}$ & $\begin{array}{c}\text { Disease } \\
\text { Duration in Yrs } \\
\text { (St. Dev })\end{array}$ \\
\hline Elderly Controls & 9 & $66.75(10.53)$ & N/A & N/A \\
\hline AD & 20 & $72.19(6.43)$ & $18.76(7.90)$ & $4.9(3.1)$ \\
\hline FTD & 26 & $63.70(10.05)$ & $19.91(6.67)$ & $3.5(2.4)$ \\
\hline
\end{tabular}

rapid sagittal T1-weighted image was acquired to align the patient in the scanner. Then, a high resolution volumetric structural scan was obtained. The volumes are comprised of T1-weighted 3D spoiled gradient echo images, with a repetition time (TR) of $35 \mathrm{~ms}$, echo time (TE) of $6 \mathrm{~ms}$, flip angle of 30 degrees, matrix size of 128 x 256 and a rectangular field of view giving an in-plane resolution of $0.9 \mathrm{x}$ $1.3 \mathrm{~mm}$, and slice thickness of $1.5 \mathrm{~mm}$. The subjects spent a total of ten minutes in the scanner to acquire the anatomical images.

Image Analysis Morphometric analysis was performed on structural images from each group using the 1999 version of the Statistical Parametric Mapping (SPM) package (Wellcome Department of Cognitive Neurology). [1] The VBM analysis was carried out as follows. All of the images were first normalized to match the SPM T1-weighted template brain. Non-linear normalization was performed with the following parameters: bilinear interpolation, $7 \times 8 \times 7$ basis functions with 12 iterations. Next, each voxel of the normalized image was classified into gray matter, white matter and cerebrospinal fluid using the SPM segmentation algorithm. Then, the segmented images were smoothed with a $12 \mathrm{~mm}$ Gaussian kernel. Two kinds of statistical analyses were performed using only the smoothed gray matter segmented images. First, a two-sample t-test was carried out comparing each patient group to the control group. Then, using a regression analysis, we correlated gray matter density to cognitive test scores for each patient group. [1]

\section{Results}

Anatomical results We used a statistical threshold of $p<0.0001$ uncorrected (equivalent to a $Z$-score of 3.82) and an extent threshold of 50 adjacent voxels. Anatomical results are summarized in Tables 2 and 3. A rendering of brain atrophy location for AD and FTD can be found in Figure 1. AD patients have significant atrophy that is most prominent in temporal cortex but also involves frontal cortex, and the atrophy is more evident in the right hemisphere than the left hemisphere. FTD patients show significant atrophy in frontal cortices, particularly in right inferior frontal and left superior frontal regions.

Atrophy-Behavior correlation results The rendered brains displaying the anatomic distribution of the correlations are found in Figure 2. In AD, performance accuracy on the confrontation naming task (54\% mean correct) correlates 
Table 2. Anatomic distribution of gray matter atrophy in AD compared to healthy control subjects.

\begin{tabular}{|l|ccc|c|c|}
\hline $\begin{array}{l}\text { Atrophy Locus } \\
\text { (Brodmann Area) }\end{array}$ & \multicolumn{2}{|l|}{ Coordinates } & $\begin{array}{c}\text { Atrophy Extent } \\
\text { (voxels) }\end{array}$ & $Z$-values \\
\hline $\begin{array}{l}\text { Right Middle Temporal, } \\
\text { Superior Temporal(BA 21,22) }\end{array}$ & 44 & -30 & 20 & 7967 & 4.97 \\
\hline $\begin{array}{l}\text { Left Middle Temporal, } \\
\text { Superior Temporal(BA 21,22) }\end{array}$ & -64 & -6 & -16 & 2145 & 4.34 \\
\hline Right Precuneus(BA 7,31) & 10 & -50 & 38 & 118 & 4.30 \\
\hline Right Middle Frontal(BA 8) & 34 & 38 & 48 & 66 & 4.21 \\
\hline Right Medial Frontal(BA 10) & 16 & 50 & 2 & 178 & 4.21 \\
\hline Right Inferior Frontal(BA 6) & 40 & 8 & 56 & 121 & 4.15 \\
\hline Right Middle Frontal(BA 46) & 54 & 34 & 22 & 68 & 4.01 \\
\hline Left Inferior Frontal(BA 45) & -40 & 14 & 14 & 141 & 3.99 \\
\hline Left Medial Temporal(BA 25) & -8 & 0 & -8 & 311 & 3.98 \\
\hline Left Fusiform(BA 20) & -50 & -32 & -24 & 354 & 3.87 \\
\hline
\end{tabular}

Table 3. Anatomic distribution of gray matter atrophy in FTD compared to healthy control subjects.

\begin{tabular}{|l|ccc|c|c|}
\hline $\begin{array}{l}\text { Atrophy Locus } \\
\text { (Brodmann Area) }\end{array}$ & \multicolumn{2}{|l|}{ Coordinates } & $\begin{array}{c}\text { Atrophy Extent } \\
\text { (voxels) }\end{array}$ & $Z$-values \\
\hline Right Inferior Frontal(BA 47) & 54 & 32 & -16 & 67 & 4.05 \\
\hline Left Parietal(BA 7) & -22 & -50 & 48 & 57 & 3.88 \\
\hline Left Middle Frontal(BA 8) & -28 & 20 & 46 & 77 & 3.81 \\
\hline
\end{tabular}

with atrophy in lateral aspects of the right temporal and parietal lobes as summarized in Table 4. Table 5 shows a correlation in FTD patients between confrontation naming accuracy (69\% mean correct) and atrophy also in the right temporal region (superior and inferior), and in the left frontal and temporal regions.

\section{Discussion}

A statistical threshold of $p<0.001$ uncorrected (equivalent to a $Z$-score of 3.1 ) and an extent threshold of 50 adjacent voxels was used in the correlation analyses. We implemented VBM analyses in a user-independent manner to quantify the anatomic distribution of atrophy in mild to moderate AD and FTD patients. We found bilateral temporal atrophy in AD, consistent with the known distribution of histopathological abnormalities in these patients. This is also consistent with previous ROI-based analyses of regional atrophy in AD. 48 VBM analyses of FTD showed atrophy in a different anatomic distribution from AD. The FTD group had more atrophy in the left hemisphere with several isolated frontal regions. These findings are also consistent in part with previous ROI based analyses, and with the known distribution of pathology in FTD. 15]13.

These different anatomic distributions of anatomy in distinct neurodegenerative diseases are thought to explain the different clinical presentations of these patients. We tested this expectation with correlations that can relate atrophy of 


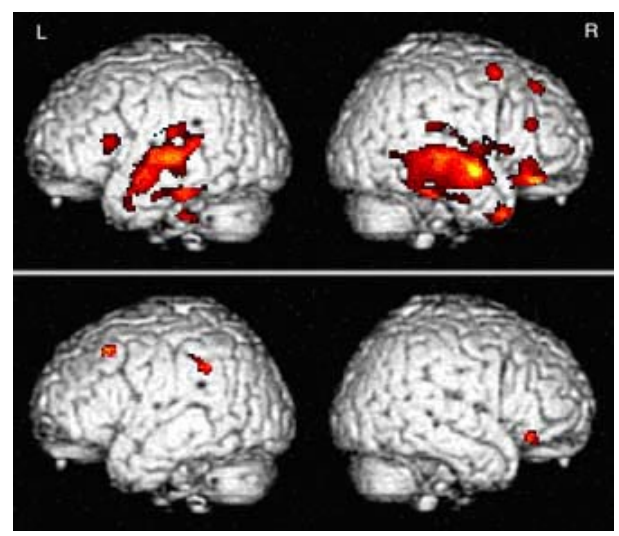

Fig. 1. Atrophy compared to control. (Top) AD. (Bottom) FTD.
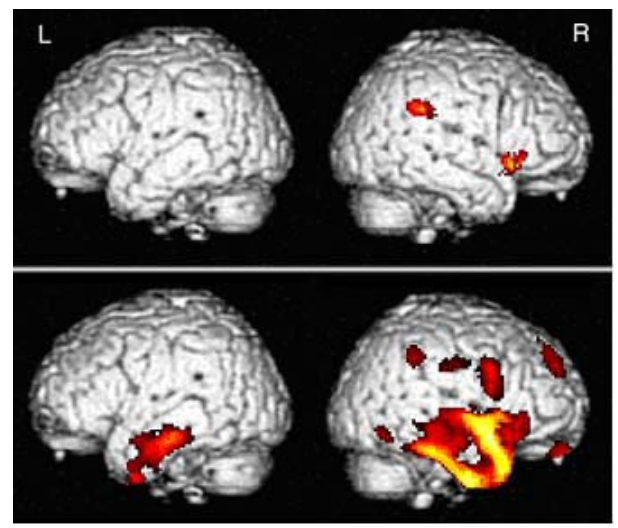

Fig. 2. Correlation of atrophy to BNT. (Top) AD. (Bottom) FTD.

these brain regions to common clinical difficulties in these patients. We examined impairments in naming to illustrate our method, though measures of semantic comprehension, executive functioning, and other cognitive measures also can be used.

In $\mathrm{AD}$, we found correlations between naming accuracy and right inferior frontal and superior regions. These are homologues of left hemisphere regions often implicated in naming in functional imaging studies of healthy adults, and we and others have found compensator up regulation of ordinarily quiescent right hemisphere regions on functional neuroimaging studies of language tasks in AD. This correlation of naming with right hemisphere structural anatomy thus may reflect the brain regions contributing to residual naming success in AD.

In fMRI activation studies of FTD, we have also found compensatory activation of brain regions not ordinarily recruited in healthy subjects to help support language functioning. We found with our automated VBM technique, naming 
Table 4. Atrophy-Behavior correlation results of AD subjects.

\begin{tabular}{|l|ccc|c|c|}
\hline $\begin{array}{l}\text { Correlation Locus } \\
\text { (Brodmann Area) }\end{array}$ & Coordinates & $\begin{array}{c}\text { Correlation Extent } \\
\text { (voxels) }\end{array}$ & $Z$-values \\
\hline Right Inferior Frontal(BA 47) & 30 & 24 & -8 & 309 & 3.22 \\
\hline Right Inferior Parietal(BA 39,40) & $44-18$ & 28 & 230 & 3.13 \\
\hline
\end{tabular}

Table 5. Atrophy-Behavior correlation results of FTD subjects.

\begin{tabular}{|l|ccc|c|c|}
\hline $\begin{array}{l}\text { Correlation Locus } \\
\text { (Brodmann Area) }\end{array}$ & Coordinates & $\begin{array}{c}\text { Correlation Extent } \\
\text { (voxels) }\end{array}$ & $Z$-values \\
\hline $\begin{array}{l}\text { Right Anterior Temporal, } \\
\text { Inferior Temporal(BA 12, 20) }\end{array}$ & 60 & 10 & -10 & 8453 & 4.95 \\
\hline $\begin{array}{l}\text { Left Inferior Temporal, } \\
\text { Middle Temporal(BA 20, 21) }\end{array}$ & -50 & -24 & -16 & 1308 & 4.50 \\
\hline Right Inferior Frontal(BA 44) & 68 & 8 & 22 & 369 & 4.29 \\
\hline $\begin{array}{l}\text { Left Middle Frontal, } \\
\text { Inferior Frontal }\end{array}$ & -24 & 28 & -8 & 195 & 3.78 \\
\hline Right Anterior Cingulate(BA 24) & 8 & 8 & 26 & 304 & 3.48 \\
\hline Right Inferior Parietal(BA 40) & 62 & -44 & 40 & 132 & 3.30 \\
\hline Right Postcentral(BA 1,2,3) & 68 & -18 & 36 & 87 & 3.27 \\
\hline Right Orbital Frontal(BA 11) & 6 & 56 & -22 & 149 & 3.22 \\
\hline Left Superior Frontal(BA 8) & -2 & 42 & 48 & 256 & 3.18 \\
\hline Right Middle Frontal(BA 9) & 22 & 50 & 34 & 169 & 3.15 \\
\hline Left Medial Temporal(BA 36) & -26 & -32 & -20 & 99 & 3.13 \\
\hline Right Inferior Temporal(BA 37) & 58 & -64 & -10 & 92 & 3.12 \\
\hline
\end{tabular}

accuracy correlates with bilateral temporal and right frontal and parietal cortices. While these areas of anatomic correlation do not necessarily correspond to the distribution of anatomic atrophy, it is likely that the connectivity pattern of cortical regions involved in naming links significantly atrophic areas with areas unusual to naming that may have less significant atrophy.

These preliminary results emphasize the feasibility of using computational morphometry to define regional gray matter atrophy in AD and FTD, and help us improve diagnostic accuracy and understand brain-behavior relationships in neurodegenerative diseases.

\section{References}

1. Ashburner J, Friston KJ. Voxel-based morphometry-The methods. Neuroimage 2000; 11:805-821.

2. Ashburner J, Friston KJ. Why voxel-based morphometry should be used. Neuroimage $2001 ; 14: 1238-1243$.

3. Baron JC, Chetelat G, Desgranges B, Perchey G, Landeau B, De la Sayette V, Eustache F. In vivo mapping of gray matter loss with voxel-based morphometry in mild Alzheimer's disease. Neuroimage 2001; 14:298-309. 
4. Bierer L, Hof P, Purohit D, Carlin L, Schmeidler J, Davis K, Perl D. Neocortical neurofibrillary tangles correlate with dementia severity in Alzheimer's disease. Archives of Neurology 1995; 52(1):81-88.

5. Bookstein, FL. "Voxel-Based Morphometry" should not be used with imperfectly registered images. Neuroimage 2001; 14:1454-1462.

6. Bozeat S, Gregory CA, Ralph MAL, Hodges JR. Which neuropsychiatric and behavioral features distinguish frontal and temporal variants of frontotemporal dementia from Alzheimer's disease? Journal of Neurology, Neurosurgery, and Psychiatry $2000 ; 69(2): 178-186$.

7. Departments of Neurology and Neuropsychology, Department of Neuroanatomy, Department of Neuroradiology. Memory disorders in probable alzheimer's disease: The role of hippocampal atrophy as shown with MRI. Journal of Neurology, Neurosurgery, and Psychiatry 1995; 58(5):590-597.

8. Eberhard DA, Lopes MBS, Trugman JM, Brashear HR. Alzheimer's disease in a case of cortical basal ganglionic degeneration with severe dementia. Journal of Neurology, Neurosurgery, and Psychiatry 1996; 60(1):109-110.

9. Frisoni GB, Beltramello A, Geroldi C, Weiss C, Bianchetti A, Trabucchi M. Brain atrophy in frontotemporal dementia. Journal of Neurology, Neurosurgery \& Psychiatry 1996; 61(2):157-165.

10. Gitelman DR, Ashburner J, Friston KJ, Tyler LK, Price CJ. Voxel-based morphometry of herpes simplex encephalitis. Neuroimage 2001; 13:623-631.

11. Good CD, Johnsrude IS, Ashburner J, Henson RNA, Friston KJ, Frackowiak RSJ. A voxel-based morphometric study of ageing in 465 normal adult human brains. Neuroimage 2001; 14:21-36.

12. Grossman M, Payer F, Onishi K, White-Devine T, Morrison D, D'Esposito M, Robinson K, Alavi A. Constraints on the cerebral basis for semantic processing from neuroimaging studies of Alzheimer's disease. Journal of Neurology, Neurosurgery, and Psychiatry 1997; 63(2):152-158.

13. Kertesz A, Munoz D. Pick's disease, frontotemporal dementia, and Pick complex: Emerging concepts. Archives of Neurology 1998; 55(3):302-304.

14. Levy M, Miller B, Cummings J, Fairbanks L, Craig A. Alzheimer disease and frontotemporal dementias: Behavioral distinctions. Archives of Neurology 1996; 53(7):687-690.

15. Lund and Manchester Groups. Clinical and neuropathological criteria for frontotemporal dementia. Journal of Neurology, Neurosurgery, and Psychiatry 1994; 57:416-418.

16. Mummery CJ, Patterson K, Wise RJS, Vandenbergh R, Price CJ, Hodges JR. Disrupted temporal lobe connections in semantic dementia. Brain 1999; 122:61-73.

17. Rozzini L, Lussignoli G, Padovani A, Bianchetti A, Trabucchi M. Alzheimer disease and frontotemporal dementia. Archives of Neurology 1997; 54(4):350-352.

18. Snowden JS, Bathgate D, Varma A, Blackshaw A, Gibbons ZC, Neary D. Distinct behavioral profiles in frontotemporal dementia and semantic dementia. Journal of Neurology, Neurosurgery, and Psychiatry 2001; 70(3):323-332.

19. Wilke M, Kaufmann C, Grabner A, Putz B, Wetter TC, Auer DP. Gray matterchanges and correlates of disease severity in schizophrenia: A statistical parametric mapping study. Neuroimage 2001; 13:814-824.

20. Yamaguchi S, Meguro K, Itoh M, Hayasaka C, Shimada M, Yamazaki H, Yamadori A. Decreased cortical glucose metabolism correlates with hippocampal atrophy in Alzheimer's disease as shown by MRI and PET. Journal of Neurology, Neurosurgery, and Psychiatry 1997; 62(6):596-600. 